\title{
Adaptive Beam Search to Enhance On-device Abstractive Summarization
}

\author{
Harichandana B S S \\ Samsung $R \& D$ Institute \\ Bangalore, India \\ hari.ss@samsung.com
}

\author{
Sumit Kumar \\ Samsung $R \& D$ Institute \\ Bangalore, India \\ sumit.kr@samsung.com
}

\begin{abstract}
We receive several essential updates on our smartphones in the form of SMS, documents, voice messages, etc. that get buried beneath the clutter of content. We often do not realize the key information without going through the full content. SMS notifications sometimes help by giving an idea of what the message is about, however, they merely offer a preview of the beginning content. One way to solve this is to have a single efficient model that can adapt and summarize data from varied sources. In this paper, we tackle this issue and for the first time, propose a novel Adaptive Beam Search to improve the quality of on-device abstractive summarization that can be applied to SMS, voice messages and can be extended to documents. To the best of our knowledge, this is the first ondevice abstractive summarization pipeline to be proposed that can adapt to multiple data sources addressing privacy concerns of users as compared to the majority of existing summarization systems that send data to a server. We reduce the model size by $30.9 \%$ using knowledge distillation and show that this model with a $97.6 \%$ lesser memory footprint extracts the same or more key information as compared to BERT.

Index Terms-On-device, Short Text Summarization, Speech
\end{abstract} Summarization, Beam Search, Knowledge Distillation

\section{INTRODUCTION}

Summarization is a significant challenge of natural language understanding [1]. There are mainly two techniques of summarization, i.e., extractive and abstractive methods. Most successful summarization systems utilize extractive approaches that crop out and stitch together portions of the source to produce a summary [2]. In contrast, abstractive summarization systems generate new phrases, possibly rephrasing or using words that were not in the original source [3]. Extractive text summarization is easier to build however summarization by abstractive technique is superior because they produce better semantically related summary [4]. Therefore, we choose abstractive summarization.

Gartner predicts that by $2022,80 \%$ of smartphones shipped will have on-device AI capabilities [5]. Though various cloudbased models are rendering domain-based summaries, most of them are not fit to be deployed on mobile devices due to their large size. Most of the current text summarization applications send the extracted text to the server to get its summarized version leading to privacy issues. On-device summarization has been explored before but most of these use extractive approach to build summaries [6] [7].

978-1-6654-4175-9/21/\$31.00 @2021 IEEE
On-device deployment is a significant challenge due to limited availability of memory and computational power [8]. However, one of the challenges with summarization itself is that it is hard to generalize. For example, summarizing a news article is very different from summarizing a financial earnings report. Certain text features like document length or genre (tech, sports, travel, etc.) make the task of summarization a serious data science problem to solve. For this reason, we aim to build an efficient model that can adapt to many sources thus enabling a single model to work for multiple use cases and saving the memory required otherwise by deploying multiple models for the same. To tackle the generalization issue, we explore solutions on the decoding side.

A popular decoding technique used by seq2seq models is Beam Search [9]. However, we find that traditional beam search does not always give consistent and meaningful results during summarization. We introduce our novel Adaptive Beam Search in Section V A significant contribution of our proposed method is summarizing not only long but also short text.

Short text (like SMS) summary is relatively unexplored as compared to long text summarization. There are multiple applications of short text summary on mobile devices and wearables to provide crisp notifications owing to their small screen size. As per a recent survey by Perficient [10] on mobile voice usage, over $19 \%$ of users prefer to dictate messages, and over 5\% users send voice messages, making speech summarization an important task to improve user experience.

In this paper, we present an abstractive on-device summarization method that can be used for a variety of mobile applications using an Adaptive Beam Search (ABS) mechanism. The main contributions of this paper are as follows:

- We propose for the first time, an on-device architecture for abstractive summarization of short text, long text, and speech using a novel adaptive Beam Search strategy.

- We propose a novel method of optimal summary selection using Beam Search.

- We reduce model size using Knowledge Distillation and evaluate its effect on model performance.

- We benchmark short text summarization performance of our model with BERT [11] and also evaluate overall text and speech summarization performance on CNN-DM [12] and How2 [13] datasets respectively. 
The experiments on our architecture show up to a $13 \%$ improvement in the number of keywords extracted in the final summary compared with BERT, and with a 97.6\% decrease in model-size comparatively.

\section{BACKGROUND}

Summarization has been used for various applications. [14] first introduced a neural attention seq2seq model with an attention-based encoder and a neural language model augmented decoder to generate an abstractive summary achieving significant improvement over conventional methods. The following years have extended the model developed using recurrent decoders, and variational autoencoders [15]. [16] used pointer networks [17] [18]. With recent advancement in the use of pre-trained language models [19] for Natural Language Processing(NLP) tasks, Bidirectional Encoder Representations(BERT) [11] was used to encode the document and generate an extractive summary by stacking several intersentence Transformer layers. [20] introduces new technique for speech summarization. Past research on Beam search include some works like [21]. There has been research done to optimize this further for the required use case In [22]. But, this increases the inference time, is not feasible to deploy on-device and does not help increase generalizability as it depends on source. Thus we propose a novel technique to overcome these issues.

\section{Traditional BeAm SEARCH}

Beam Search [9] is a search strategy that tries to choose better results from all possible candidates efficiently. We observe that the traditional Beam Search strategy does not always yield semantically correct summaries. We experimented using a pointer-generator network [16] with the coverage and traditional Beam Search with a beam width equal to 4 (determined after experimentation to optimize inference time) on short text (SMS) data. The results are shown in Table I] It is seen that this often results in misleading summaries when used on data other than news (as it is trained only on news data CNN-DM, as mentioned in Section VI-A). For example, in the third example in Table I, the summary does not describe the actual message and is misleading. To improve the performance we experiment using a pointer-generator with better Beam Search strategies.

\section{SEARCh Strategies EXPERIMENTED}

Since we aim to create a single model that can generalize across varied types of sources, we experiment with a strategy in which source-specific keywords are given more priority by increasing the log-probabilities of all hypotheses containing these according to their category. To obtain these keywords, we created an in-house dataset consisting of source files in the form of SMS, and documents belonging to different categories (OTPs, travel, etc.) (categories were determined through an internal survey). The dataset consists of 100 files of each category collected from $>100$ individuals from diverse backgrounds. We consider the top 30 most frequently occurring words after removing stop words and common english words as keywords. Dataset details are shown in Table II

\section{A. Keyword based step wise increment}

First, we experimented using a pointer-generator with a keyword-based log-probability increment at each step. Depending on the category of the document, a list of keywords

TABLE I

SMS SUMMARIZATION RESULTS USING DIFFERENT VARIANTS OF BEAM SEARCH HIGHLIGHTING WRONG TOKENS IN RED AND CORRECTLY IDENTIFIED KEY POINTS IN BLUE

\begin{tabular}{|c|c|c|c|c|}
\hline Source & $\begin{array}{l}\text { Taditional Beam } \\
\text { Search }\end{array}$ & $\begin{array}{l}\text { Beam Search with } \\
\text { keyword based step- } \\
\text { wise increment }\end{array}$ & $\begin{array}{l}\text { Beam Search with } \\
\text { keyword based end } \\
\text { increment }\end{array}$ & $\begin{array}{l}\text { Adaptive Beam } \\
\text { Search (ABS) }\end{array}$ \\
\hline $\begin{array}{l}\text { Dear Member, EPF Contribution of Rs. } \\
13458 \text { against UAN } 100385505604 \text { for } \\
\text { due month } 122016 \text { has been received. } \\
\text { Passbook will be updated shortly. Re- } \\
\text { gards EPFO }\end{array}$ & $\begin{array}{l}\text { passbook } \\
\text { contribution of } \\
\text { rs. } 13458 \text { against uan } \\
100385505604 \text { for } \\
\text { due }\end{array}$ & $\begin{array}{l}\text { passbook } \\
\text { contribution of } \\
\text { rs. } 13458 \text { against uan } \\
100385505604 \text { for } \\
\text { due }\end{array}$ & $\begin{array}{l}\text { passbook } \\
\text { contribution of } \\
\text { rs. } 13458 \text { against uan } \\
100385505604 \text { for d }\end{array}$ & $\begin{array}{l}\text { dear member, epf } \\
\text { contribution of rs. } \\
13458 \text { against uan }\end{array}$ \\
\hline $\begin{array}{l}\text { Buy Gated Farmhouse near Bangalore } \\
\text { @ } 12.5 \text { Lakhs } 45 \text { Min frm Airport on } \\
\text { NH } 7 \text { Developed, Maintained and Af- } \\
\text { fordable Excellent Appreciation What- } \\
\text { sApp: trkk.in/Ua/3mR }\end{array}$ & $\begin{array}{l}45 \text { min frm airport on } \\
\text { nh } 7 \text {. }\end{array}$ & $\begin{array}{l}\text { lakhs / ua/3mr buy } \\
\text { gated farmhouse near } \\
\text { bangalore @ } 12.5 \\
\text { lakhs. }\end{array}$ & $\begin{array}{l}\text { buy gated farmhouse } \\
\text { near bangalore @ } \\
12.5 \text { lakhs. devel- } \\
\text { oped, maintained }\end{array}$ & $\begin{array}{l}\text { buy gated farmhouse } \\
\text { near bangalore @ } \\
12.5 \text { lakhs . devel- } \\
\text { oped, maintained }\end{array}$ \\
\hline $\begin{array}{l}\text { Txn of INR } 209.00 \text { done on Acct } \\
\text { XX013 on 15-DEC-19.Info: VPS*KAI } \\
\text { P.Avbl Bal:INR 50,698.72.Call } \\
\text { 18002662 for dispute or SMS BLOCK } \\
013 \text { to } 9215676766\end{array}$ & $\begin{array}{l}\text { call } 18002662 \text { for dis- } \\
\text { pute or sms block } 013 \\
\text { to } 9215676766 \text {. }\end{array}$ & $\begin{array}{l}\text { txn * kai p.avbl : inr } \\
\text { acct . call } 18002662 \\
\text { for dispute }\end{array}$ & $\begin{array}{l}\operatorname{txn} 50,698.72 \text { of inr } \\
209.00 \text { done on acct } \\
x x 013 \text { on } 15 \text {-dec-19. }\end{array}$ & $\begin{array}{l}\text { txn of inr } 209.00 \\
\text { done on acct xx013 } \\
\text { on } 15-\text { dec- } 19 . \text { info }\end{array}$ \\
\hline
\end{tabular}


TABLE II

IN-HOUSE DATASET DETAILS

\begin{tabular}{l|l}
\hline Source & Categories \\
\hline SMS & $\begin{array}{l}\text { Travel tickets, Bank transactions, verification } \\
\text { code, advertisements, miscellaneous }\end{array}$ \\
\hline Documents & $\begin{array}{l}\text { Travel, Personal information, Receipts, pa- } \\
\text { pers/books, Miscellaneous }\end{array}$ \\
\hline
\end{tabular}

was determined which needs to be given higher weightage during summarization as mentioned above. In this model, we increase the log-probabilities of these words at each step in the Beam Search decoder according to Eq. (1) where $T_{i j}$ is the log-probabilities generated by the decoder at step $i$ and for $j^{t h}$ word token in the vocabulary. After thorough experimentation, we determine the optimal value of $\alpha$ to be 0.1 . But since this strategy is biased towards maximum keyword occurrence irrespective of semantic correctness, this leads to incorrect sentence formations (in the 2nd example in Table I the word 'lakhs' is generated twice).

$$
P_{i j}=T_{i j} * \alpha
$$

\section{B. Keyword based end hypothesis increment}

Next, we increase the log-probabilities of keywords only at the final step of Beam Search for all the end hypotheses instead of at each step according to the (11). This gives better results than the previous strategy, but errors persist (in 1st example, "passbook contribution" is predicted instead of "EPF contribution" which is wrong). To solve these issues, we propose a novel strategy of using an Language Model (LM) with Beam Search.

\section{Proposed Adaptive Beam SeArch}

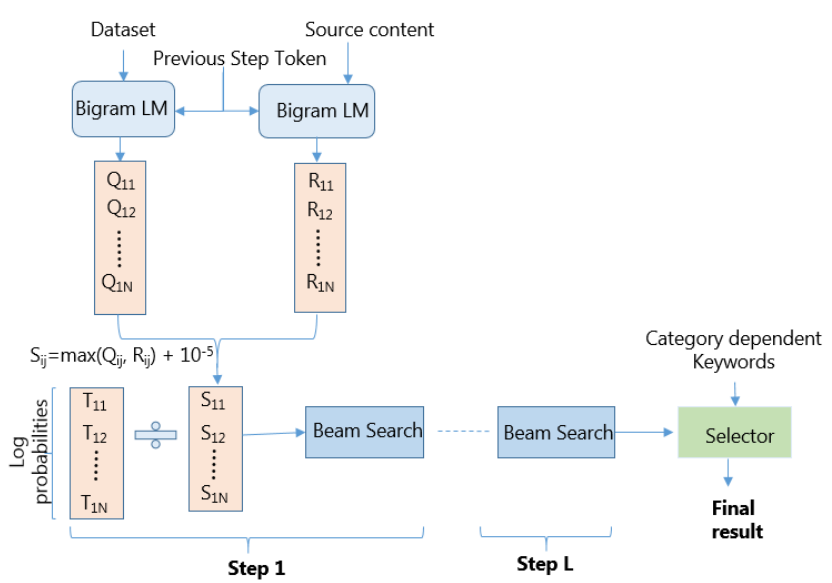

Fig. 1. Adaptive Beam Search for optimized summarization

To solve the issue of inconsistent summaries generated through the above search strategy, we use a bigram language model to improve the quality of the final summary generated and also propose a new scoring strategy to select the best hypothesis at the end.

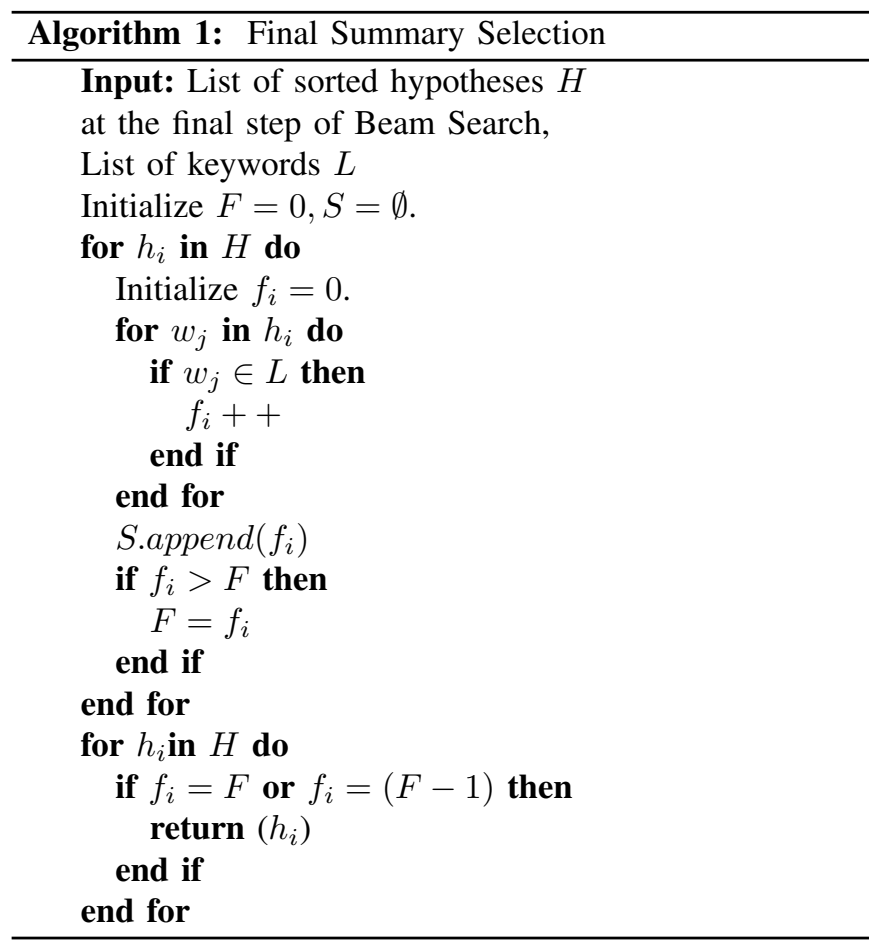

We enhance the Beam Search by connecting a bigram language model shown in Fig. 11. The dataset given as input to the language model is different for each category of text source. The model calculates the probability of bigram occurrence which is then added to the $\log$ probabilities from the decoder as per Eq. (2)

$$
P_{i j}=\frac{T_{i j}}{\left(\max \left(Q_{i j}, R_{i j}\right)+10^{-5}\right)}
$$

where $Q_{i j}$ is the bigram probability of token predicted in the $(i-1)^{t h}$ step and the $j^{t h}$ token from vocabulary using input dataset. In case $i$ is 1 , then the 'start' token is considered as the previous step token. Similarly, $R_{i j}$ is calculated using bigrams taken from the source content and $T_{i j}$ is the logprobabilities generated by the decoder at step $i$ and for $j^{t h}$ word token in the vocabulary. We use the source bigrams to consider cases like bank statements where the amount is unique in each transaction. Thus, this captures the grammatical correctness of bigrams in source content even if it is not seen previously in the dataset.

We add a small quantity of $10^{-5}$ to avoid division by 0 . The Selector uses the method described in Algorithm 1 to select the final summary. $L$ is the list of common and category-specific keywords (explained in Section IV-A) for the given source and $H$ is the list of all hypotheses ( $h_{i}$ where $0<i \leq$ beam width) sorted according to the average log-probabilities in the last step of Beam Search. Each hypothesis $h_{i}$ contains $j$ word tokens represented by $w_{j} . F$ is the global maximum frequency of keywords (in $L$ ) considering all hypotheses in $H$ i.e, $F=$ 
$\max \left(f_{i}\right) \forall f_{i} \in S$ where $S$ is the set of all $f_{i}$, the individual keyword frequency of $h_{i}$. We select the first hypothesis with $f_{i}=F$ or $f_{i}=(F-1)$ as the final summary. We have included (F-1) hypotheses also to allow other summaries with a significant number of keywords with higher log-probability to get chosen. The length of the final summary is dependent on the length of the source text content. As it is found that users would make the majority of decisions using the longer levels of summary (15\% and 30\%) according to [23], after experimenting, we found $15 \%$ to be optimal. Thus, we use the minimum length to be $15 \%$ of the source length and the maximum length to be $35 \%$ of the source length. Also, the length of the summary is one of the parameters that can be configured by the user (To enable flexible summary size).

\section{Overall Pipeline}

From low complexity simple rule-based extractive models to heavily parameterized Transformer based abstractive models, there are various approaches for summarizing text. To choose the most optimal approach for on-device summarization is a challenge as the requirements and environment during deployment can be significantly different from those during the development phase as stated in [24]. There exists a tradeoff between model performance and model size. The pointergenerator network showed promising results on summarization and deploying this architecture on-device is plausible as the model size is relatively small, due to which we experiment on architectures using this as the base. The detailed architecture is explained in the following section.

\section{A. Dataset}

The model is first trained on CNN-DM dataset [12] [25] which contains online news articles. The pretrained model is then trained on How2 Dataset [13] for speech summarization.

\section{B. Preprocessing}

We use simple text pre-processing where the words are first converted to lower case and the special characters are separated. The words are then tokenized and indexed according to the vocabulary (which is of size $50 \mathrm{~K}$, determined to optimize final model size). With a batch size of 16 , each article is padded to ensure that the input is of the same length and then fed to the model.

\section{Adaptive Beam Search (ABS)}

We use our proposed ABS strategy along with pointergenerator network [16] to decode during inferencing to adapt to the type of data source. We make use of source-specific LM to enable the model to adapt to the source the details of which are explained in Section VII

\section{Knowledge Distillation}

Knowledge distillation(KD) [26] is a technique to compress large models. We experiment to perform KD on our model by using a temperature of 1 . Considering $\alpha$ to be equal to $1-\beta$ the final loss function for the student model is described in (3).

$$
\text { Loss }=(1-\beta) * \text { Loss }_{\text {target }}+\beta * \text { Loss }_{t-s}
$$

Loss $_{t-s}$ is the cross-entropy loss of the student and teacher distributions and Loss target is the cross-entropy loss considering target summary. We consider the value of $\beta$ to be 0.4 as our intention was for the model to be slightly more biased towards the ground truth. The teacher architecture consists of 256 BiLSTM cells, and embedding dimension as 128 with a total of 400 steps. We use a decoder consisting of 100 steps for training. In student architecture, we reduce the number of BiLSTM cells to 150 . We train the student directly, using a teacher model trained with coverage loss which excludes the need to train the student with coverage loss again. The final training pipeline is shown in Figure 2

\section{E. On-device deployment}

For deploying on-device, we first performed quantization using TensorFlow tflite as well as graph transform tool on the student model to further reduce the size, but we observed a significant deterioration in the performance. Thus, we do not use the quantized model. We implement preprocessing and beam search decoding on Android as the model graph after freezing does not support these operations. We build the TensorFlow [27](version 1.10) library from source including some operations previously not included(like ScatterND) in the default shared object file and use the built shared object file to facilitate model inference on-device.

\section{SUMMARIZATION ON DIFFERENT SOURCES}

This model architecture supports the summarization of SMS, voice messages and can be extended for documents and URLs

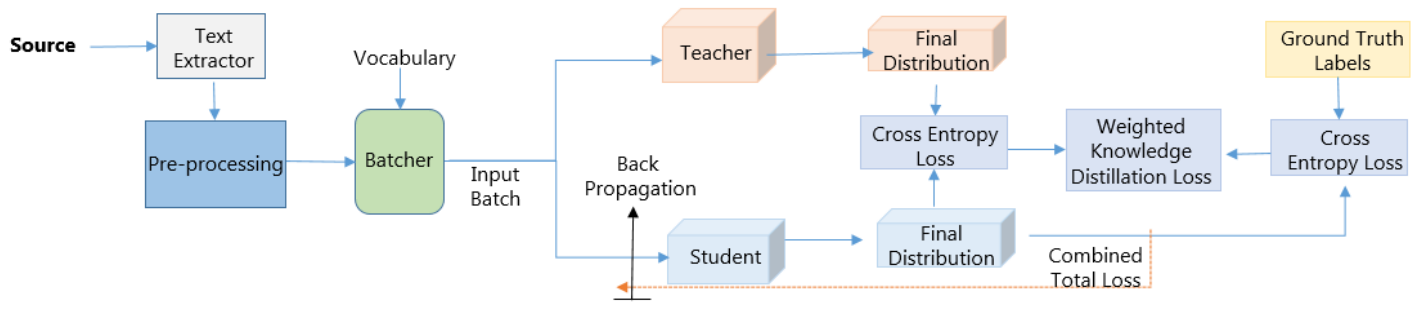

Fig. 2. Pipeline used for training the final summarization model 
TABLE III

SMS SUMMARIZATION RESULTS BENCHMARKED WITH BERT RESULTS HIGHLIGHTING KEY TOKENS (IN BLUE) NECESSARY IN SUMMARY

\begin{tabular}{|c|c|c|c|}
\hline Source & $\begin{array}{l}\text { Pointer-Generator with } \\
\text { ABS }\end{array}$ & $\begin{array}{l}\text { Pointer-Generator with } \\
\text { ABS after KD }\end{array}$ & BERT \\
\hline
\end{tabular}

TABLE IV

Comparative On-Device Performance analysis

\begin{tabular}{l|l|l|l}
\hline Model & $\begin{array}{l}\text { Keywords Re- } \\
\text { called }\end{array}$ & Model Size & $\begin{array}{l}\text { On-device } \\
\text { Inference } \\
\text { Time (sec- } \\
\text { onds/character) }\end{array}$ \\
\hline $\begin{array}{l}\text { Pointer genera- } \\
\text { tor }\end{array}$ & $49 \%$ & $80.07 \mathrm{MB}$ & $0.035 \mathrm{~s} / \mathrm{char}$ \\
\hline BERT & $56 \%$ & $>2 \mathrm{~GB}$ & $-^{*}$ \\
\hline $\begin{array}{l}\text { Pointer genera- } \\
\text { tor with ABS } \\
\text { after KD }\end{array}$ & $69 \%$ & $55.3 \mathrm{MB}$ & $0.03 \mathrm{~s} / \mathrm{char}$ \\
\hline
\end{tabular}

TABLE V

AvERAGE ROUGE F-SCORES ON CNN-DM DATASET.

\begin{tabular}{l|l|l|l}
\hline Model & ROUGE-L & ROUGE-1 & ROUGE-2 \\
\hline Teacher & $30.12 \pm 0.4$ & $33.48 \pm 0.4$ & $13.41 \pm 0.4$ \\
\hline $\begin{array}{l}\text { Student before } \\
\text { KD }\end{array}$ & $28.11 \pm 0.4$ & $31.58 \pm 0.4$ & $11.43 \pm 0.3$ \\
\hline $\begin{array}{l}\text { Student after } \\
\text { KD }\end{array}$ & $30.18 \pm 0.5$ & $33.55 \pm 0.5$ & $13.36 \pm 0.4$ \\
\hline
\end{tabular}

as well. For SMS summarization, the LM is trained on SMS data categorized into travel tickets, bank transactions, OTP, advertisements, miscellaneous. Important details that need to be extracted depend upon the category of the SMS. We list out keywords like 'dollars', 'rupees', 'amount' to be common keywords across all categories and convey important details of the message in most cases. Other category-specific keywords like 'PNR', 'Delayed', etc. are only considered when the SMS belongs to a specific category (in this case 'travel') which is determined by another list of keywords. To select the final summary we use Algorithm 1 .

For speech summarization, we convert speech to text using ASR, followed by sentence boundary detection, the output is sent to the model which is also trained on the How2 dataset.
TABLE VI

Average ROUGE score evaluation on How2 Dataset.

\begin{tabular}{l|l|l|l}
\hline Metric & ROUGE-L & ROUGE-1 & ROUGE-2 \\
\hline F-score & $29.95 \pm 0.4$ & $36.54 \pm 0.4$ & $15.13 \pm 0.4$ \\
Recall & $33.46 \pm 0.5$ & $41.29 \pm 0.5$ & $17.26 \pm 0.3$ \\
Precision & $27.31 \pm 0.4$ & $34.27 \pm 0.4$ & $14.12 \pm 0.4$ \\
\hline
\end{tabular}

\section{RESULTS AND DISCUSSION}

We use the Samsung Galaxy A50 smartphone model (Android 9.0, 6GB RAM, 64GB ROM, Samsung Exynos 7 Octa 9610) to conduct our experiments.

To evaluate on SMS summarization, we benchmark the results with that of BERT (12-layers, 768-hidden, 12-heads, $110 \mathrm{M}$ parameters). We create an in-house dataset explained in IV-A and use this to calculate the accuracy of keywords extracted in the summaries by each model. We evaluate by calculating the recall of keywords in the final summary. We observe that our model can extract an average of $69 \%$ keywords as compared to $56 \%$ accuracy of BERT and $49 \%$ of pointer-generator without ABS. Some examples are as shown in Table III. The overall performance can be seen in Table IV which shows our model performs better than BERT and baseline model. The inclusion of an LM trained on the same structured text and inclusion of category-specific keywords improves the performance.

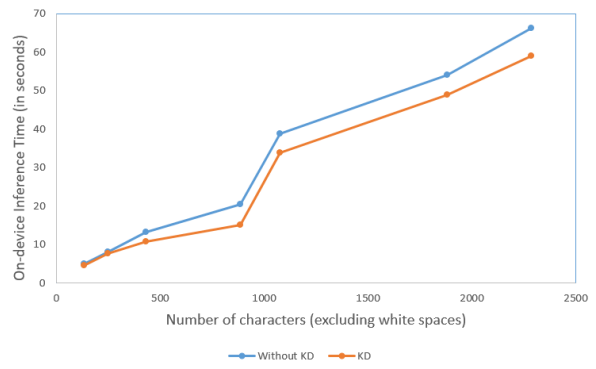

Fig. 3. Graph between inference time and character count 
To evaluate the impact of Knowledge Distillation (KD), we calculate the ROUGE scores on 4000 randomly sampled test articles of CNN-DM dataset on the teacher, student model trained without and with $\mathrm{KD}$, the F-scores of which are as shown in Table $\mathrm{V}$. The results prove that there is a strong improvement in the model performance after KD. We observe a reduction in model size by $30.9 \%$ after KD.

Fig. 3 shows the on-device inference time as a function of the character count for the model trained without and with $\mathrm{KD}$. The latter has better inference time (reduction in model loading time: $60.5 \%$, average inference time: $12.66 \%$ ).

To further check the efficacy of the student model after KD on speech, we also evaluated the performance of our model on the How2 dataset as shown in Table VI.
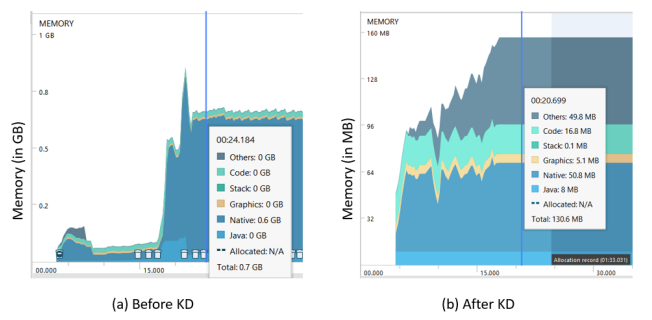

Fig. 4. Comparision between RAM used before and after KD

There is a significant reduction in on-device RAM usage as well which is measured using Android Memory Profiler. The model utilizes a total RAM of 700MB before KD which is reduced to $130.6 \mathrm{MB}$ after KD. Fig. 4 captures the RAM usage for two scenarios: with and without KD. The memory segments are captured at a particular instant of time while on-device summarization is in progress for the same sample.

The above results prove that our model adapts to short text, long text, and speech with the help of ABS.

\section{CONCLUSION AND FUtURE WORK}

In this paper, we propose a novel Adaptive Beam Search strategy and a new scoring technique for optimal on-device summarization. The proposed model can adapt to different types of text and speech sources on-device which makes it highly useful to mobile users over the globe. We show that our model extracts more keywords as compared to BERT by $13 \%$ with a $97.6 \%$ decrease in model size. We also show that Knowledge Distillation helps reduce the model size by $30.9 \%$. In the future, it will be intriguing to explore other model compression techniques and test their efficiency for this task.

\section{REFERENCES}

[1] F. Jacquenet, M. Bernard, and C. Largeron, "Meeting summarization, a challenge for deep learning," in International Work-Conference on Artificial Neural Networks. Springer, 2019, pp. 644-655.

[2] R. Nallapati, F. Zhai, and B. Zhou, "Summarunner: A recurrent neural network based sequence model for extractive summarization of documents," in Thirty-First AAAI Conference on Artificial Intelligence, 2017.

[3] S. Chopra, M. Auli, and A. M. Rush, "Abstractive sentence summarization with attentive recurrent neural networks," in Proceedings of the 2016 Conference of the North American Chapter of the Association for Computational Linguistics: Human Language Technologies, 2016, pp. 93-98.
[4] N. Munot and S. S. Govilkar, "Comparative study of text summarization methods," International Journal of Computer Applications, vol. 102, no. 12, 2014.

[5] Gartner, "Gartner Highlights 10 Uses for AI-Powered Smartphones," https://www.gartner.com/en/newsroom/press-releases/201803-20-gartner-highlights-10-uses-for-ai-powered-smartphones 2018 , [Online; accessed 15-October-2020].

[6] L. Cabral, R. Lima, R. Lins, M. Neto, R. Ferreira, S. Simske, and M. Riss, "Automatic summarization of news articles in mobile devices," in 2015 Fourteenth Mexican International Conference on Artificial Intelligence (MICAI). IEEE, 2015, pp. 8-13.

[7] R. Chongtay, M. Last, and B. Berendt, "Responsive news summarization for ubiquitous consumption on multiple mobile devices," in 23rd International Conference on Intelligent User Interfaces, 2018, pp. 433437.

[8] X. Dai, I. Spasic, S. Chapman, and B. Meyer, "The state of the art in implementing machine learning for mobile apps: A survey," 032020.

[9] A. Graves, "Sequence transduction with recurrent neural networks," arXiv preprint arXiv: 1211.3711, 2012.

[10] E. Enge, "Mobile Voice Usage Trends in 2020," https: //www.perficient.com/insights/research-hub/voice-usage-trends 2020, [Online; accessed 15-October-2020].

[11] J. Devlin, M.-W. Chang, K. Lee, and K. Toutanova, "Bert: Pre-training of deep bidirectional transformers for language understanding," arXiv preprint arXiv: 1810.04805, 2018.

[12] K. M. Hermann, T. Kocisky, E. Grefenstette, L. Espeholt, W. Kay, M. Suleyman, and P. Blunsom, "Teaching machines to read and comprehend," in Advances in neural information processing systems, 2015, pp. 1693-1701

[13] R. Sanabria, O. Caglayan, S. Palaskar, D. Elliott, L. Barrault, L. Specia, and F. Metze, "How2: a large-scale dataset for multimodal language understanding," arXiv preprint arXiv:1811.00347, 2018.

[14] A. M. Rush, S. Chopra, and J. Weston, "A neural attention model for abstractive sentence summarization," arXiv preprint arXiv:1509.00685, 2015.

[15] Y. Miao and P. Blunsom, "Language as a latent variable: Discrete generative models for sentence compression," arXiv preprint arXiv:1609.07317, 2016.

[16] A. See, P. J. Liu, and C. D. Manning, "Get to the point: Summarization with pointer-generator networks," arXiv preprint arXiv:1704.04368, 2017.

[17] O. Vinyals, M. Fortunato, and N. Jaitly, "Pointer networks," in Advances in neural information processing systems, 2015, pp. 2692-2700.

[18] K. Xu, J. Ba, R. Kiros, K. Cho, A. Courville, R. Salakhudinov, R. Zemel, and Y. Bengio, "Show, attend and tell: Neural image caption generation with visual attention," in International conference on machine learning, 2015, pp. 2048-2057.

[19] X. Zhang, F. Wei, and M. Zhou, "Hibert: Document level pre-training of hierarchical bidirectional transformers for document summarization," arXiv preprint arXiv:1905.06566, 2019.

[20] T. Liu, S. Liu, and B. Chen, "A hierarchical neural summarization framework for spoken documents," in ICASSP 2019 - 2019 IEEE International Conference on Acoustics, Speech and Signal Processing (ICASSP), 2019, pp. 7185-7189.

[21] M. Freitag and Y. Al-Onaizan, "Beam search strategies for neural machine translation," arXiv preprint arXiv:1702.01806, 2017.

[22] X. Chen, J. Li, and H. Wang, "Keyphrase guided beam search for neural abstractive text summarization," in 2019 International Joint Conference on Neural Networks (IJCNN), 2019, pp. 1-9.

[23] S. Sweeney and F. Crestani, "Effective search results summary size and device screen size: Is there a relationship?" Information processing \& management, vol. 42, no. 4, pp. 1056-1074, 2006.

[24] L. Lai and N. Suda, "Rethinking machine learning development and deployment for edge devices," arXiv preprint arXiv:1806.07846, 2018

[25] R. Nallapati, B. Zhou, C. Gulcehre, B. Xiang et al., "Abstractive text summarization using sequence-to-sequence rnns and beyond," arXiv preprint arXiv:1602.06023, 2016.

[26] G. Hinton, O. Vinyals, and J. Dean, "Distilling the knowledge in a neural network," arXiv preprint arXiv:1503.02531, 2015.

[27] Y. T. Google Inc., "Tensorflow," https://github.com/tensorflow/ tensorflow 2016, [Online; accessed 19-August-2019]. 